\title{
Correlative Imaging and Bio-inspiration: Multi-scale and Multi-modal Investigations of the Acorn Barnacle (Semibalanus balanoides)
}

\author{
Ria L. Mitchell ${ }^{1}$, Cameron Pleydell-Pearce ${ }^{1}$, Mark P. Coleman ${ }^{1}$, Peter Davies ${ }^{1}$, Laura North ${ }^{1}$, Richard E. \\ Johnston $^{1}$ and Will Harris ${ }^{2}$ \\ 1. Advanced Imaging of Materials (AIM) Facility, College of Engineering, Swansea University, \\ Swansea, UK. \\ 2. Carl Zeiss X-ray Microscopy, Pleasanton, CA, USA.
}

Natural-forming biomaterials can be subject to extremes of moisture, chemistry, loading, temperature flux, and predation. Yet, those forming in the marine environment for example, form in ambient conditions using only the habitat-dependent resources available to the organism (e.g., elemental composition of seawater for precipitation of $\mathrm{CaCO}_{3}$ hard parts). Through evolution and adaptation, organisms have developed an incredibly diverse range of strategies to survive despite these unpredictable habitatdependent conditions; this includes the development of complex chemical-biological-structural relationships [1-2], which not only enhance structural integrity but also make the most of the resources available to them. Studying these relationships is pivotal to developing bio-inspiration strategies that can contribute to novel human-made engineering and material science development and innovation.

The process of formation and ultimately function can be better understood by investigating these complex biological systems at various length scales and linking chemical and physical properties using correlative imaging. Correlative imaging is useful because it assesses how features at the millimeter scale are related to features at the nanometer scale (and vice-versa), and how multiple modalities can be linked (e.g., physical-chemical-mechanical properties) [3-4]. Correlative imaging allows us to identify sites of interest across various 2D and 3D imaging and analytical platforms by combining light, electron and X-ray microscopy to generate diverse information taken directly from nature.

Here, we have used the correlative potential of numerous coupled systems at different length scales: Xray microscopy $(\mu \mathrm{CT})$, scanning electron microscopy (SEM), optical light microscopy $(\mathrm{OM})$, and focused ion beam microscopy (FIB) to ascertain the chemical composition, microstructural properties, and crystallographic orientation of plate joints (ala) in the 'shell' of the barnacle Semibalanus balanoides. The ala are the interlocking meeting point of neighboring plates [5] and are often 'shared' between adjoining organisms. The interlocks enable the organism to expand in size during growth, and consequently need to be structurally strong to resist damage [5]. $\mu \mathrm{CT}$ reveals complex interactions and relationships between neighboring plates that can only be identified via non-destructive 3D methods. OM and SEM indicate specific chemical and crystallographic orientations at the ala tips, which has implications for natural strengthening mechanisms and potential biologically-mediated precipitation of specific elements. FIB milling is used to isolate micro-scale areas of interest from larger macro-scale structures in order to link overall properties to microstructural feature. From this work we have developed a better understanding of how even small-scale and often overlooked organisms have mechanical and structural properties which can reveal naturally-occurring bio-engineering. Understanding the organism's behavior is essential to understand the functions of these complex forms. The work demonstrates that correlative methods that span different platforms and techniques such as this enable the extension of the fundamental basics of material science to broaden the application of bio-inspiration in human-made engineering practices. 
References:

[1] L North et al, APL Materials, 5 (2017), p. 1.

[2] JHG Rocha et al, Bone 37 (2005), p. 850.

[3] K Grandfield, H Engqvist, Advances in Materials Science and Engineering 2012 (2015), p. 1.

[4] J Gelb, T Volkenandt, A Merkle, Microscopy and Microanalysis Conference Proceedings 23 (1) (2017).

[5] GR Murdock, JD Currey, Biological Bulletin 155 (1978), p. 169.
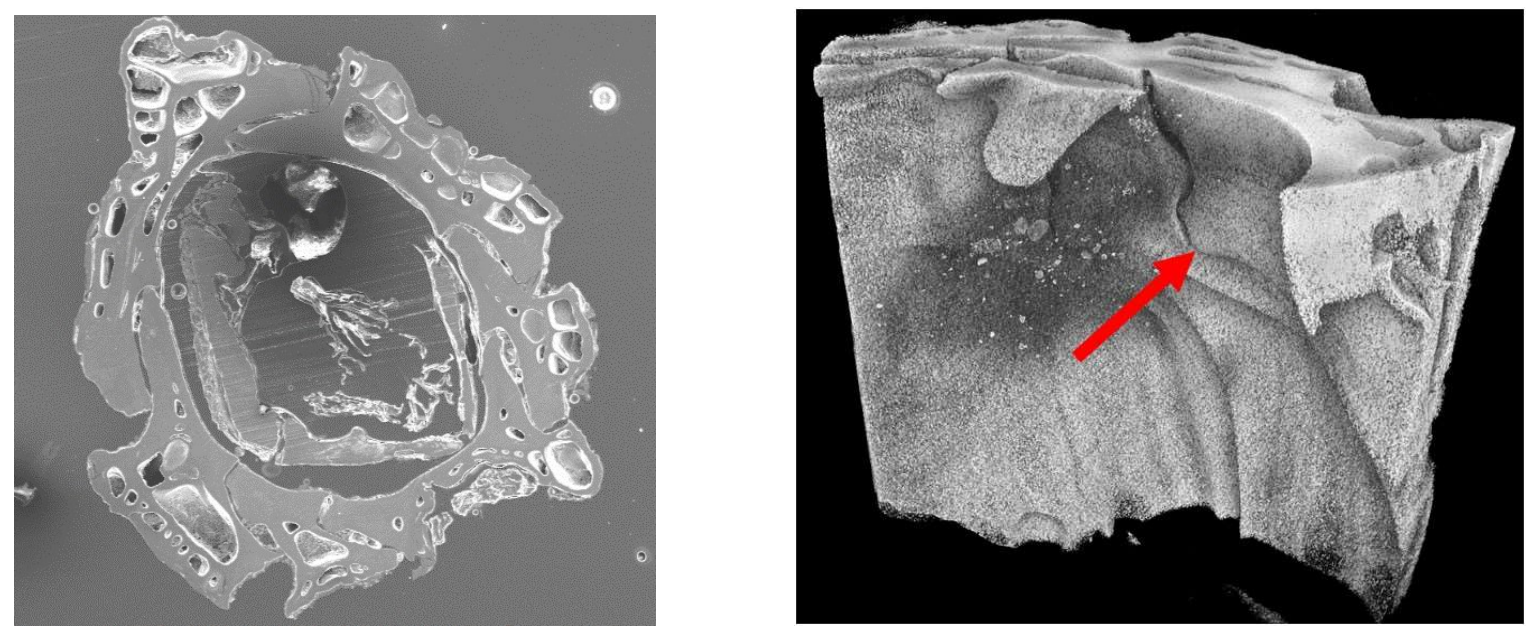

Figure 1. a) 2D SEM image of section of barnacle shell, illustrating six ala. b) $\mu \mathrm{CT}$ image of barnacle interlocking plates. Arrow indicates location of ala. This enables us to trace the ala through the 3D structure of the barnacle.

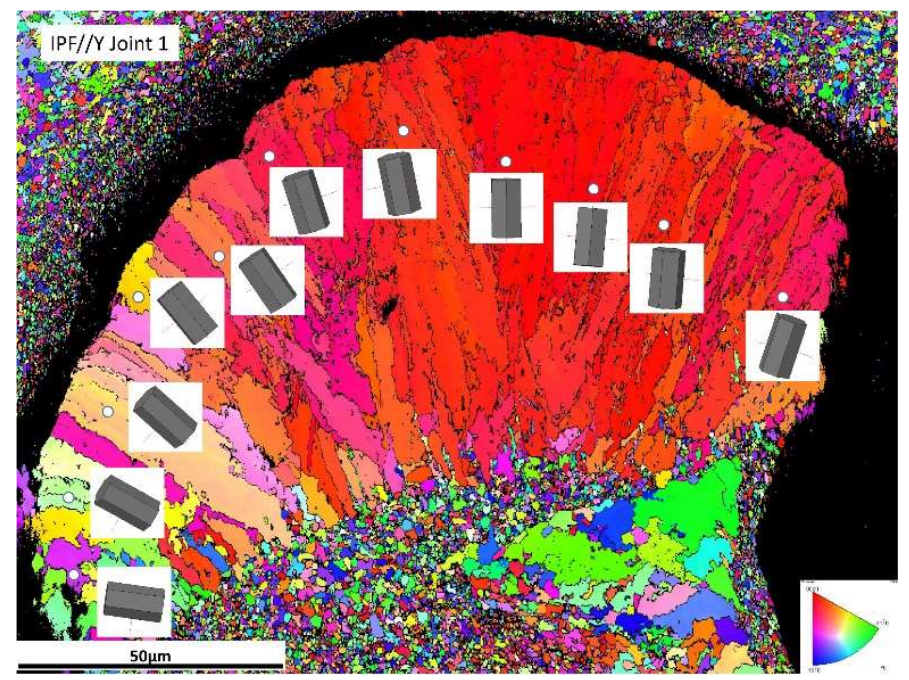

Figure 2. 2D EBSD crystallographic orientations in the tip of the ala. There is an elongation of microstructure around the tip as well as some preferred crystallographic texture, which contributes towards structural integrity. 